Taxonomía y sistemática

\title{
Una especie nueva de Epicauta de Venezuela y comentarios sobre el grupo de especies de Epicauta (E.) vittata (Coleoptera: Meloidae)
}

\author{
A new species of Epicauta from Venezuela with comments on the Epicauta (E.) vittata species group \\ (Coleoptera: Meloidae)
}

\author{
Mario García-París ${ }^{a}$, José L. Ruiz ${ }^{b}$, Alberto Sánchez-Vialas ${ }^{c}$ y E. Karen López-Estrada ${ }^{c} * *$ \\ ${ }^{a}$ Museo Nacional de Ciencias Naturales, MNCN-CSIC, c/ José Gutiérrez Abascal 2, 28006 Madrid, España \\ b Instituto de Estudios Ceutíes, Paseo del Revellín 30, Apartado postal 593, 51080 Ceuta, España

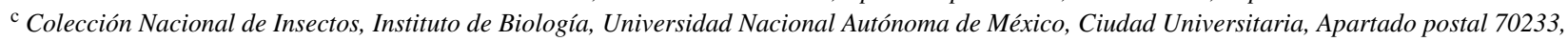 \\ 04510 Ciudad de México, México
}

Recibido el 13 de noviembre de 2015; aceptado el 20 de enero de 2016

Disponible en Internet el 18 de agosto de 2016

\begin{abstract}
Resumen
El examen de ejemplares del género Epicauta de la colección del Museo del Instituto de Zoología Aplicada (MIZA, Venezuela) nos permitió detectar la presencia de ejemplares procedentes de los estados de Lara y Falcón, con características morfológicas singulares. Estos rasgos no permiten su asignación a ninguna de las especies conocidas del género por lo que se describe como una especie nueva: Epicauta falcolarandina sp. nov., que se incluye en el grupo de especies de Epicauta vittata. E. falcolarandina se diferencia de las demás especies venezolanas del grupo de E. vittata (Epicauta aragua y Epicauta apure) por presentar la lámina inferior de las uñas de los tarsos curvada y casi de la misma anchura y longitud que la lámina superior. El patrón característico de coloración de E. falcolarandina, que recuerda al de Epicauta leopardina, la diferencia de las restantes especies del grupo de E. vittata. Una característica peculiar de esta especie, que comparte con Epicauta abadona, es que el color de la pilosidad no coincide con el de la coloración tegumentaria; así, en la mayor parte de los élitros, incluidas casi todas las zonas oscuras, la pilosidad es amarillo-blanquecina.

Derechos Reservados ( 2016 Universidad Nacional Autónoma de México, Instituto de Biología. Este es un artículo de acceso abierto distribuido bajo los términos de la Licencia Creative Commons CC BY-NC-ND 4.0.
\end{abstract}

Palabras clave: Coleoptera; Meloidae; Epicauta falcolarandina sp. nov.; Biodiversidad; Taxonomía; América del Sur; Falcón; Lara

\begin{abstract}
The study of specimens of Epicauta held at the collection of the Museo del Instituto de Zoología Aplicada (MIZA, Venezuela) allowed us to discover specimens with singular morphological traits from the states of Lara and Falcón. The characters shown by these specimens do not permit to assign them to any of the known species of the genus, and thus we consider that they represent a new species: Epicauta falcolarandina sp. nov. The new species is included in the Epicauta vittata species group. E. falcolarandina differs at first glance from all other Venezuelan species of the E. vittata species group (Epicauta aragua and Epicauta apure) because it presents the ventral blade of the tarsal claws curved and of similar width and length than the dorsal one. The characteristic coloration pattern of E. falcolarandina, which vaguely resembles the pattern displayed by Epicauta leopardina, differs from all other species of the E. vittata species group. A particular trait of E. falcolarandina, shared with Epicauta abadona, is that the coloration of the pilosity does not match the color of the tegument. Most of the surface of the elytra of E. falcolarandina, including almost all dark zones, is covered by a whitish-yellow pilosity.

All Rights Reserved (C 2016 Universidad Nacional Autónoma de México, Instituto de Biología. This is an open access item distributed under the Creative Commons CC License BY-NC-ND 4.0.
\end{abstract}

Keywords: Coleoptera; Meloidae; Epicauta falcolarandina sp. nov.; Biodiversity; Taxonomy; South America; Falcón; Lara

\footnotetext{
* Autor para correspondencia.

Correo electrónico: lokaren21@gmail.com (E.K. López-Estrada).

La revisión por pares es responsabilidad de la Universidad Nacional Autónoma de México.
} 


\section{Introducción}

El género Epicauta Dejean, 1834 es uno de los clados más diversos de la familia Meloidae. La diversificación del género alcanza su máximo en el continente americano, donde aproximadamente viven el $75 \%$ de las 361 especies conocidas. A pesar de esta enorme diversificación, en la actualidad solo se reconocen 2 subgéneros: Macrobasis LeConte, 1862, prácticamente restringido a la mitad septentrional de América, con unas pocas especies que penetran en América meridional, y Epicauta, que se extiende a lo largo de toda la distribución del género (Pinto, 1991; Pinto y Bologna, 1999). Dentro del subgénero Epicauta la separación de faunas entre América del Sur y del Norte es casi completa, ya que solo poseen en común taxones de 3 grupos de especies: el grupo de Epicauta carmelita (Haag-Rutenberg, 1880), predominantemente sudamericano que penetra con 2 especies hasta el sur de México, el grupo de Epicauta caustica Rojas, 1857, también sudamericano, que alcanza con una especie en Panamá y el grupo de Epicauta vittata (Fabricius, 1775), bien representado desde la Argentina hasta Canadá (Adams y Selander, 1979; Pinto, 1991).

La fauna de Epicauta del subgénero nominal ha sido revisada exhaustivamente en América septentrional (Pinto, 1991; García-París y Ruiz, 2013). Por el contrario, la fauna de Epicauta de América meridional no ha sido objeto de ninguna revisión de conjunto y la mayor parte de las especies descritas no han sido asignadas a ningún grupo de especies concreto. Los grupos de E. vittata, E. caustica, Epicauta bella Mäklin, 1875 y Epicauta maculata (Say, 1824) que fueron revisados por Adams y Selander (1979), Selander (1981), Campos-Soldini (2011) y Campos-Soldini y Roig-Juñent $(2011,2015)$, representan las excepciones a esta situación. Entre ellos, la taxonomía del grupo de E. vittata es todavía y a pesar de la revisión de Adams y Selander (1979) y Campos-Soldini y Roig-Juñent (2011), una de las más complejas en el ámbito de Epicauta americanas. Pinto (1991) basándose en el estudio de las especies norteamericanas, caracteriza al grupo de E. vittata por los siguientes rasgos: especies de tamaño medio, con una longitud que varía desde los 7 a los $22 \mathrm{~mm}$; élitros con un patrón característico de líneas claras (amarillo-anaranjadas) y oscuras (negras o pardo oscuras) que se manifiestan tanto a nivel del tegumento como en la pilosidad; ojos anchos, salientes, marcadamente emarginados que se extienden hacia la zona media de la cabeza más que en los demás grupos de especies; pronoto relativamente estrecho y alargado con su máxima anchura en el tercio apical; antenas delgadas, adelgazando progresivamente hacia el ápice con o sin dimorfismo sexual, si lo presentan, los machos pueden tener los artejos antenales comprimidos o carenados; tibias anteriores de los machos pueden presentar uno o 2 espolones apicales; el posterior cuando existe es recto, el anterior puede ser recto o curvado ventralmente; esternito abdominal VI del macho suele estar ligeramente emarginado en el ápice; parámeros de la genitalia masculina incompletamente esclerotizados excepto a lo largo de una estrecha banda media que se bifurca y recorre la zona media del área ventral de cada parámero. Las larvas del primer estadio de las especies conocidas del grupo presentan reticulaciones tegumentarias proyectadas hacia atrás en la porción anterior y posterior de los terguitos I a VII; las sedas terminales del tercer segmento antenal son cortas ( 3 cuartos de la longitud de las del segmento II como máximo); los palpos labiales poseen 2-3 sedas en el segmento II; y la cara ventral del fémur anterior presenta 8 sedas lanceoladas (Pinto, 1991).

Adams y Selander (1979) incluyeron inicialmente en el grupo 31 especies, 27 de ellas descritas por autores anteriores a su revisión. Ellas son Epicauta abadona Skinner, 1904, Epicauta aemula (Fischer, 1827), Epicauta borgmeieri Denier, 1935, Epicauta bosqi Denier, 1935, Epicauta clericalis (Berg, 1881), Epicauta floydwerneri Martínez, 1955, Epicauta franciscana Denier, 1935, Epicauta fuliginosa (Olivier, 1895), Epicauta grammica (Fischer, 1827), Epicauta kraussi (Haag-Rutenberg, 1880), Epicauta leopardina (HaagRutenberg, 1880), Epicauta luteolineata (Pic, 1933), Epicauta missionum (Berg, 1881), Epicauta monachica (Berg, 1883), Epicauta nattereri (Haag-Rutenberg, 1880), Epicauta occidentalis Werner, 1944, Epicauta philaemata (Klug, 1825), Epicauta purpureiceps (Berg, 1889), Epicauta rutilifrons Borchmann, 1930, Epicauta strigata (Gyllenhal, 1817), Epicauta subvittata (Erichson, 1848), Epicauta unilineata Champion, 1892, E. vittata (Fabricius, 1775), Epicauta vitticollis (Haag-Rutenberg, 1880), Epicauta xanthocephala (Klug, 1825), Epicauta yungana Denier, 1935 y Epicauta zebra (Dorhn, 1876), y otras 4 especies que describieron en dicho trabajo: Epicauta apure, Epicauta aragua, Epicauta tamara y Epicauta temexa, las 2 primeras de Venezuela y las otras 2 de México, aunque con una distribución geográfica más amplia. Adams y Selander (1979) consiguieron resolver definitivamente la confusión secular con respecto a las especies del grupo en la región oriental de los Estados Unidos, ya clarificada en parte por Werner (1944), que implicaba a $E$. vittata y sus 2 subespecies (E. vittata vittata y E. vittata lemniscata Fabricius, 1801), E. occidentalis y E. temexa. Por otra parte, la descripción de E. aragua resuelve el problema de las especies centroamericanas, integrado por esta y por $E$. vitticollis y E. unilineata, sin embargo, la descripción de E. apure complica la situación taxonómica de las especies sudamericanas que presentan la lámina inferior de las uñas de los tarsos recta y más larga que la lámina superior, como E. grammica.

A partir de esta revisión los cambios taxonómicos son escasos. Agafitei y Selander (1980) describieron las larvas de varias especies del grupo reefiniendo los caracteres del grupo. Selander (1981) transfirió E. floydwerneri al grupo de E. caustica. Pinto (1991) señaló que posiblemente E. tamara y E. unilineata representen extremos de la variabilidad de una única especie. García-París, Buckle y Parra-Olea (2007) reconocieron la validez nomenclatural de Epicauta dugesi Werner, 1957, que al tener prioridad sobre E. tamara permite establecer la sinonimia de esta última con E. dugesi. Campos-Soldini (2011) transfirió E. zebra al grupo de E. bella. Campos-Soldini y Roig-Juñent (2011) redefinen el grupo de E. vittata interpretando de forma restrictiva los caracteres indicados por Pinto (1991). De esta forma incluyen en el grupo de $E$. vittata exclusivamente a aquellas especies que presentan un patrón de coloración tegumentaria listado (con independencia de la pilosidad) y cuyos machos presentan los parámeros parcialmentte sin esclerotizar. Utilizando estos criterios, Campos-Soldini y Roig-Juñent (2011) incluyen por vez 
primera en el grupo de E. vittata a Epicauta excavata (Klug, 1825) y a Epicauta semivittata (Fairmaire, 1875), mientras que excluyen a E. borgmeieri, E. franciscana, E. kraussi, E. philaemata, E. purpureiceps, E. yungana y E. zebra. Todas las especies excluidas por Campos-Soldini y Roig-Juñent (2011) permanecen sin asignar a ningún grupo de especies, con excepción de $E$. zebra, que asignaron al grupo de E. bella.

Tras estos cambios, el grupo quedaba conformado por 22 especies, 14 de ellas localizadas exclusivamente en América del Sur, 7 en América del Norte y una, E. aragua, con poblaciones extendidas tanto al norte del istmo de Panamá como al sur del mismo. Es muy posible que como indica Pinto (1991), el grupo incluya alguna especie más de las ya descritas de la fauna sudamericana. Las relaciones internas dentro del grupo son desconocidas y según Agafitei y Selander (1980), las larvas proporcionan poca información filogenética a ese nivel.

Durante la revisión preliminar del material de Epicauta de la colección del Museo del Instituto de Zoología Aplicada (MIZA) de la Universidad Central de Venezuela en Maracay, efectuada con el objeto de determinar la distribución geográfica de las especies venezolanas y de aportar caracteres que permitan clarificar la situación taxonómica de E. apure, localizamos 2 hembras de una especie desconocida del grupo de $E$. vittata, que por sus rasgos externos no puede asignarse a ninguna de las especies conocidas y que por su característico patrón de coloración se asemeja lejanamente a E. leopardina, una especie de amplia distribución en el centro del subcontinente sudamericano (Adams y Selander, 1979).

En este artículo describimos esta especie nueva del grupo de $E$. vittata localizada en las zonas subáridas del noroeste venezolano y comentamos de forma general los problemas taxonómicos que presenta el grupo, ya que su resolución sobrepasa el ámbito geográfico del estudio aquí iniciado y requiere el examen de amplias series de material de toda América meridional.

\section{Materiales y métodos}

La descripción de la especie nueva de Epicauta está basada en el estudio de 2 ejemplares, ambos hembra, colectados en 2 localidades de zonas subáridas del noroeste de Venezuela, los cuales se encuentran depositados en el Museo del Instituto de Zoología Agrícola de la Universidad Central de Venezuela (Maracay, Venezuela). La adscripción de la especie al grupo de E. vittata se basa en los caracteres que proporcionan Adams y Selander (1979), Pinto (1991) y Campos-Soldini y Roig-Juñent (2011). Como material de comparación de los taxones del grupo en el que se integra la nueva especie, se han estudiado ejemplares de las especies que se indican a continuación y cuyos datos se relacionan en el apéndice: E. abadona, E. apure, E. aragua, E. dugesi, E. excavata, E. grammica, E. leopardina, E. luteolineata, E. missionum, E. monachica, E. occidentalis, E. semivittata, E. temexa, E. unilineata, E. vittata y E. vitticollis. Las instituciones $\mathrm{y}$ colecciones de las que se ha estudiado material de comparación y sus respectivas siglas son las siguientes: Colección Nacional de Insectos del Instituto de Biología de la Universidad Nacional Autónoma de México (CNIN-IBUNAM), Ciudad de México; Estación de Biología de Chamela (EBCH) del Instituto de Biología de la Universidad Nacional Autónoma de México, Jalisco, México; El Colegio de la Frontera Sur (ECOSUR), San Cristóbal de las Casas, México; Hungarian Natural History Museum (HNHM), Magyar Természettudományi Múzeum, Budapest, Hungría; Colección M. A. Bologna (MAB), Universita Roma Tre, Roma, Italia; Museo del Instituto de Zoología Agrícola (MIZA) de la Universidad Central de Venezuela, Maracay, Venezuela; Natural History Museum (NHM), Londres, UK; Natural History Museum of Los Angeles County (NHMLA), California, Estados Unidos.

Las comparaciones con respecto a E. aemula, E. bosqi, E. clericalis, E. fuliginosa, E. nattereri, E. subvittata, y E. rutilifrons se realizaron a partir de la descripción original de la especie o de descripciones posteriores cuando existen. No se efectuaron comparaciones con E. borgmeieri, E. franciscana, E. kraussi, E. philaemata, E. purpureiceps, E. yungana y E. zebra, excluidas del grupo de $E$. vittata por Campos-Soldini y Roig-Juñent (2011), que por otra parte presentan patrones de coloración muy distintos a los de la nueva especie.

El estudio morfológico se efectuó mediante estereomicroscopio. La medida de la longitud del pronoto se tomó desde el nivel del extremo de los lóbulos del margen posterior hasta el punto medio del margen anterior y la medida de la anchura pronotal corresponde a la máxima entre los márgenes laterales. Estas medidas se tomaron con micrómetro acoplado a uno de los oculares. Las fotografías se efectuaron a ejemplares secos con cámara digital y con microscopio electrónico ambiental (Philips Quanta 200).

\section{Descripción}

Epicauta (Epicauta) falcolarandina sp. nov. García-París, Ruiz, Sánchez-Vialas y López-Estrada

Holotipo (figs. 1a, b y 2): una hembra, con etiqueta blanca parcialmente impresa: «Parapara/ Venezuela, Lara/ 22-24-XI1974»; etiqueta blanca parcialmente impresa: «E. Osuna/ F. Cerda./D. Grance»; etiqueta roja, impresa, «Holotypus/ E. (Epicauta) falcolarandinal García-París, Ruiz, Sánchez-Vialas et López-Estrada des. 2016». Paratipo (fig. 1c, d): una hembra, etiqueta blanca impresa: «Venezuela Falcón/ Sanare. Finca Ti-/ llerias 100 m./ 27 al 30-X-1978»; etiqueta blanca impresa: «J. Clavijo./ A. Chacon./ et al.»; etiqueta roja, impresa, «Paratypus/ E. (Epicauta) falcolarandinal García-París, Ruiz, SánchezVialas et López-Estrada des. 2016».

Descripción del holotipo: aspecto general estilizado y grácil. Longitud total: $12.9 \mathrm{~mm}$; anchura máxima, situada un poco antes del ápice elitral: $4.4 \mathrm{~mm}$. Cabeza, pronoto, élitros y patas con tegumento mate, de color ocre-amarillento con manchas y bandas oscuras más o menos extensas y límites poco definidos de color pardo achocolatado (fig. 1a, b). Antenas y región ventral de color pardo rojizo con zonas difusas oscurecidas. Pilosidad general amarillo blanquecina.

Cabeza de anchura similar a la de la base del pronoto, de $1.7 \mathrm{~mm}$ desde la sutura fronto-clipeal hasta el vértex y $1.9 \mathrm{~mm}$ de anchura entre las sienes. Sienes subparalelas, redondeadas hacia el vértex, de $0.7 \mathrm{~mm}$ de longitud (medida en visión lateral 


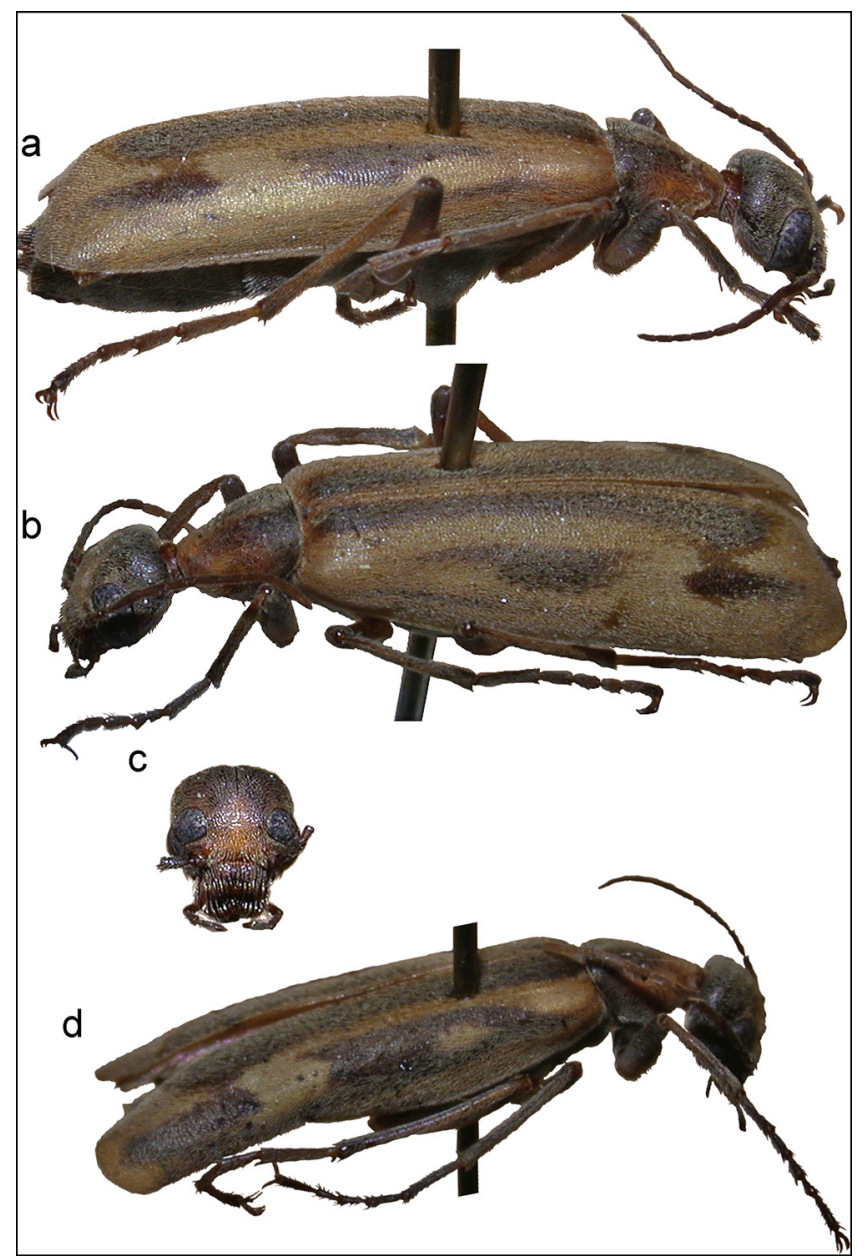

Figura 1. Habitus de Epicauta falcolarandina. a) y b) Vistas laterales del holotipo. c) Vista frontal de la cabeza (paratipo). d) Vista lateral del paratipo. Nótense las diferencias en la amplitud y distribución de la coloración de los élitros y de la pilosidad elitral.

desde el margen posterior del ojo hasta el extremo de las mismas; figura 1c). Ojos grandes, de $1.15 \mathrm{~mm}$ de diámetro mayor y $0.65 \mathrm{~mm}$ de diámetro menor, prominentes, de contornos redondeados, rodeados por un reborde marcado y brillante, con una amplia escotadura interna que los divide en 2 lóbulos, el inferior de mayor tamaño y prolongado hasta la base de las maxilas. Distancia mínima entre ojos: $0.95 \mathrm{~mm}$. Frente plana, de color ocre amarillento que se va transformando en pardo oscuro hacia las sienes y el vértex, con 2 elevaciones suaves situadas junto al borde interno del lóbulo superior del ojo y con un surco central, liso, fino y poco marcado que se extiende desde el centro de la frente hasta el vértex. Inserciones de las antenas apenas levantadas (figs. 1c, 2b). Sutura clípeo-frontal arqueada. Clípeo subrectangular, transverso, de $0.95 \mathrm{~mm}$ de ancho y $0.5 \mathrm{~mm}$ de largo, con el tercio anterior membranoso y liso, de color pardo rojizo (fig. 1c). Labro transverso, fuertemente bilobulado, con escotadura central bien marcada, de $0.9 \mathrm{~mm}$ de anchura máxima y $0.4 \mathrm{~mm}$ de longitud máxima, de color pardo obscuro en la región basal y pardo rojizo en la distal. Mandíbulas de color castaño con el ápice casi negro, fuertemente curvadas en su borde externo, que apenas sobresalen por debajo del labro.
Punteado de la cabeza fino y muy denso, casi confluente, uniformemente repartido, un poco más denso en el disco de la frente, menos denso en el extremo posterior de las sienes y ausente en 2 pequeñas áreas lisas justo por encima de las inserciones de las antenas. Punteado del clípeo denso, constituido por puntos aproximadamente una vez y media más gruesos que los de la frente; zona membranosa sin puntos. Punteado del labro con los puntos similares a los del clípeo aunque un poco más impresos, distribuidos por los 2 tercios posteriores. Pilosidad de la cápsula cefálica densa, siguiendo el patrón del punteado en el que se inserta, constituida por pelos cortos blanquecino-amarillentos tumbados y aplicados contra el tegumento, dirigidos hacia delante. Clípeo con pilosidad casi el doble de larga que la de la frente, formada por pelos amarillo-blanquecinos semierectos y dirigidos hacia adelante, que sigue el patrón de los puntos en los que se inserta. Labro con pilosidad como la del clípeo, un poco más corta y más dispersa. Márgenes externos de la mitad proximal de las mandíbulas con algunos pelos un poco más cortos y tumbados que los del labro. Palpos maxilares alargados con el artejo basal (I) estrecho, subcilíndrico y muy corto; artejo II alargado, troncocónico con la máxima anchura en el extremo, 2.2 veces más largo que ancho; artejo III alargado, troncocónico, con la máxima anchura en el ápice, un poco más corto que el II, 1.7 veces más largo que ancho; artejo IV alargado, subtriangular, con la mayor anchura en el extremo, 2.2 veces más largo que ancho, ligeramente deprimido dorsoventralmente y con el ápice parcialmente truncado en oblicuo; en el ápice se marca una hendidura que deja al descubierto una zona sensorial; la pilosidad de los 3 últimos artejos constituida por pelos cortos, blanquecinoamarillentos similares a los del margen de las mandíbulas excepto el tercio apical del último artejo que es glabro (fig. 2c, d). Palpos labiales muy cortos con el último artejo subtriagular, fuertemente dilatado en el extremo, casi igual de ancho que de largo, casi glabros, con algunos pelos dispersos finos y cortos.

Antenas alargadas, de aspecto filiforme, extendidas hacia atrás sobrepasan ligeramente la región humeral de los élitros, de color castaño con el primer artejo algo más oscuro y los 4 últimos más claros; artejo I corto, troncocónico, con la máxima anchura en el ápice, 2.3 veces más largo que ancho y 2.2 veces más largo que el segundo; artejo II corto, subcilíndrico, 1.25 veces más largo que ancho; artejo III subcilíndrico, ligeramente comprimido lateralmente, 2.8 veces más largo que el II y 1.4 veces más largo que el IV; artejos IV a VII subiguales, de lados paralelos, ligeramente comprimidos lateralmente, aproximadamente 2.5 veces más largos que anchos; artejos VII-IX del mismo aspecto que los anteriores pero ligeramente más cortos; artejo XI subcilíndrico fusiforme, redondeado en su extremo, 1.4 veces más largo que el X. Artejos I y II con pilosidad blanquecinoamarillenta, semierecta, similar a la de las sienes; artejo III con pilosidad blanquecino-amarillenta tumbada, más fina, corta y densa que los de los artejos I y II; artejos IV al XI con pilosidad amarillo-dorada, cortísima densa y aplicada contra el tegumento. Artejos III al XI con 5 a 8 pelos erectos, finos, de longitud similar a los del artejo III, amarillo-dorados distribuidos irregularmente por la superficie de los artejos.

Pronoto de $2 \mathrm{~mm}$ de largo y $1.85 \mathrm{~mm}$ de anchura máxima, situada a nivel de la base (fig. 2a); de color ocre-amarillento 


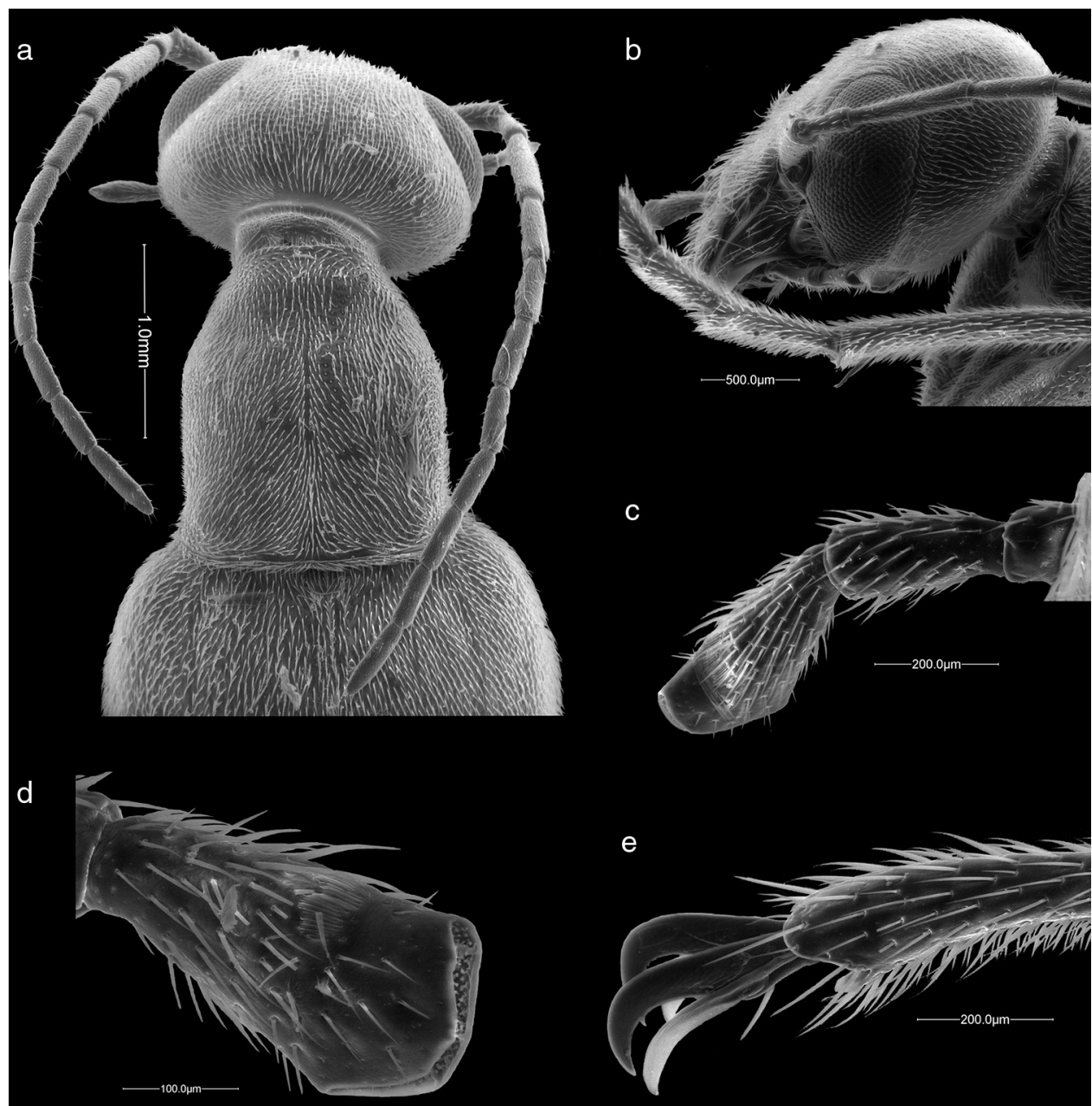

Figura 2. Rasgos anatómicos de Epicauta falcolarandina. a) Vista dorsal del pronoto y cabeza del holotipo. b) Vista lateral de la cabeza. c) y d) Detalle de los palpos labiales. e) Uñas del último segmento tarsal, nótese la curvatura de la lámina inferior.

en la zona central de la mitad anterior, en la mitad anterior de los márgenes laterales y en una fina banda difusa central en la región posterior, con un sombreado pardo chocolate en la mitad posterior que se extiende hacia delante en 2 bandas que rodean a la región ocre-amarillenta central y alcanzan el margen anterior; campaniforme y alargado con los lados subparalelos en los 2 tercios posteriores y convergentes en el tercio anterior; con una depresión central leve y difusa junto a la base; márgenes anterior y posterior con un reborde fino que recorre toda su longitud; superficie dorsal ligeramente en declive hacia delante, sin surco medio longitudinal. Punteado fino y denso, casi confluente y de aspecto rasposo, uniformemente repartido. Pilosidad densa, corta y fina, blanquecino-amarillenta, similar a la de la frente, aplicada sobre el tegumento y dirigida hacia atrás, excepto en una estrecha porción basal donde se dirige hacia los lados; la de las propleuras dirigida hacia arriba; margen posterior con una línea de pelos semierectos de longitud similar a los de la superficie del pronoto, oblicuamente dirigidos hacia los lados, excepto en los ángulos posteriores donde se dirigen hacia dentro. Escutelo pequeño, hemielíptico, de color pardo-chocolate, cubierto por pilosidad densa, similar a la de los élitros.
Élitros alargados con lados divergentes hacia atrás, dehiscentes en el quinto apical y redondeados distalmente; cada uno de $4.5 \mathrm{~mm}$ de longitud y $2.3 \mathrm{~mm}$ de máxima anchura en la zona posterior; con un leve pliegue longitudinal más cercano a la sutura que al margen externo, más marcado en el tercio anterior y otro similar más cercano a la sutura que parte de la base del húmero; húmeros redondeados. Coloración de base ocreamarillento con un patrón de manchas y líneas pardo-chocolate de límites difusos que consiste en: una banda longitudinal que se extiende junto a la sutura desde la base del élitro hasta el sexto posterior y que se ensancha cerca del final para acercarse a otra mancha longitudinal ligeramente más externa, corta y de forma alargada-elipsoidal, de aspecto más oscuro al estar cubierta por pilosidad castaño oscura; una tercera banda longitudinal de anchura irregular alineada con la anterior, que recorre el élitro en su tercio central; una línea gruesa longitudinal muy próxima al margen lateral, apenas visible desde arriba, que se extiende desde la región subhumeral hasta la mitad del élitro y por último, una estrecha lúnula apical ligeramente oscurecida (fig. 1a, b). Punteado muy fino, denso y uniformemente distribuido, de aspecto rasposo. Pilosidad elitral densa, muy corta, semitendida, de color amarillento blanquecino a excepción de la 
mancha posterior externa que presenta pilosidad de color castaño oscuro, así como de algunos pelos oscuros, casi negros, escasos y dispersos, distribuidos por toda la superficie del élitro. Prosterno, mesosterno y metasterno de color castaño, con las regiones centrales ligeramente aclaradas, con punteado muy fino y denso y pilosidad blanquecino-plateada, de aspecto similar a la del pronoto aunque un poco más larga. Alas claras, traslúcidas, con la vena costal oscurecida.

Patas con los trocánteres y 2 tercios proximales de los fémures de color ocre amarillento, el tercio apical de los fémures, tibias y tarsos castaño, más o menos oscurecido; de aspecto grácil y alargadas; con densa pilosidad similar a la de los élitros. Tibias con 2 espinales apicales, las de las tibias anteriores y medias rectas, cortas, delgadas y terminadas en punta aguda, la interna de las tibias posteriores similar a aquellas pero un poco más ancha, la externa más ancha, un poco más corta, algo curvada y cortada en bisel en el ápice; cara interna del fémur anterior con una profunda escotadura que presenta pelos rígidos, cortos, dorados y apretados en su región posterior, siendo la región anterior lisa y glabra; esta escotadura femoral encaja en una muesca de la protibia que también porta pelos dorados a los de dicha escotadura. Tarsos alargados, un poco más largos que las tibias correspondientes, estrechos, casi filiformes, con cepillos de pelos amarillo-dorados cortos, muy apretados y erectos que cubren toda la cara inferior del tarso, excepto una fina línea longitudinal media. Uñas tarsales con el lóbulo superior fuertemente curvado y el inferior ligeramente más estrecho $\mathrm{y}$ algo menos curvado aunque de longitud similar al superior (fig. 2c).

Esternitos abdominales de aspecto lustroso y color pardo rojizo un poco más oscurecidos en los márgenes posteriores; punteado muy fino y denso, rasposo aunque poco impreso; pilosidad fina, corta y densa de color blanquecino plateado; último esternito abdominal con el ápice redondeado. Pigidio similar a los esternitos abdominales, con una estrecha hendidura central en su margen posterior.

\section{Resumen taxonómico}

Variabilidad: macho desconocido. El único paratipo estudiado, también hembra, presenta un patrón de coloración similar al del holotipo, aunque con las áreas oscuras mucho más extensas: antenas, cabeza excepto la región anterior de la frente, clípeo, labro y palpos, patas excepto la mitad proximal de los fémures, prosterno, mesosterno y metasterno y esternitos abdominales pardo chocolate; pronoto y élitros con áreas oscuras más extendidas, de manera que en los élitros son más anchas y contactan parcialmente entre sí (fig. 1d). La longitud del paratipo es $11.4 \mathrm{~mm}$.

Etimología: el adjetivo «falcolarandina» se refiere a la distribución geográfica conocida de la especie, limitada a los estados de Lara y Falcón, en el norte de Venezuela.

Distribución geográfica: los 2 únicos ejemplares se colectaron en zonas semiáridas de los estados de Falcón y Lara. El aislamiento geográfico de esta peculiar y fitoclimáticamente característica región de Venezuela ha sido puesto de manifiesto en numerosos estudios (Mijares-Urrutia y Arends, 2000; LemusJiménez y Ramírez, 2002) y el número de especies endémicas de grupos diversos que alberga es muy elevado (Mijares-Urrutia y Arends, 2001; Vázquez, Madi, Cordero y Rodríguez, 2011; Wuster, Yrausquin y Mijares-Urrutia, 2001). Aunque las especies del grupo de $E$. vittata parecen mostrar áreas de distribución relativamente grandes, es muy posible que dadas las caracterísitcas de la región donde se ha localizado, E. falcolarandina sea una especie endémica de esta región árida.

\section{Comentarios taxonómicos}

E. falcolarandina se integra en el subgénero Epicauta sensu Pinto (1991) por presentar los caracteres siguientes: peine de las tibias posteriores ausente; segundo artejo de las antenas corto, siempre menos largo que la mitad del tercero; primer artejo de las antenas sin excavaduras ni fosas apicales, generalmente más corto que el tercero y con la superficie posterior recta, sin arquear (Pinto, 1991). Pinto indica además como carácter diferenciador del subgénero Epicauta, el ojo débilmente emarginado con el lóbulo dorsal más largo que el ventral, sin embargo, el grupo de $E$. (E.) vittata constituye una excepción, pues la mayor parte de las especies que lo integran (incluida E. falcolarandina) presentan la emarginación bien marcada y el lóbulo inferior del ojo de mayor tamaño que el superior. La monofilia de los subgéneros de Epicauta necesita ser evaluada en un contexto filogenético que incluya representantes del Viejo Mundo.

Dentro del subgénero Epicauta, E. falcolarandina se encuadra en el grupo de $E$. vittata, por presentar el patrón de coloración tegumentaria elitral característico de las especies del grupo, en el que alternan líneas o bandas claras amarillo-anaranjadas y oscuras que se manifiesta tanto a nivel del tegumento como en la pilosidad, aunque no necesariamente de forma coincidente (Pinto, 1991; Campos-Soldini y Roig-Juñent, 2011). Además, tal como señala Pinto (1991), para las especies del grupo, estas presentan los ojos anchos, marcadamente emarginados y extendidos hacia la zona media de la cabeza y el pronoto relativamente estrecho y alargado, de lados subparalelos hasta el cuarto anterior a partir de donde convergen progresivamente. El resto de caracteres del grupo se refieren exclusivamente a características de los machos que no podemos contrastar en E. falcolarandina.

E. falcolarandina se diferencia al primer examen de las demás especies de Epicauta venezolanas del grupo de E. vittata (E. aragua y E. apure) por presentar la lámina inferior de las uñas de los tarsos curvada y aproximadamente de la misma longitud y anchura que la lámina superior (fig. 2e), mientras que en $E$. aragua y en $E$. apure, la lámina inferior es casi recta y claramente más fina que la superior. Este mismo carácter la separa de $E$. subvittata y de E. grammica. Además, E. aragua, E. apure y E. grammica poseen una coloración muy distinta, con la cabeza rojiza, no tan oscura como en E. falcolarandina y el tegumento en general mucho más oscuro, con las bandas negras de los élitros ocupando casi toda la superficie elitral excepto en 2 finas líneas longitudinales, a menudo fusionadas en la zona humeral.

El patrón característico de coloración de E. falcolarandina la diferencia de las restantes especies del grupo de E. vittata, que por lo general muestran patrones relativamente constantes dentro de cada zona geográfica. Una característica peculiar de esta especie es que el color de la pilosidad no coincide con el 


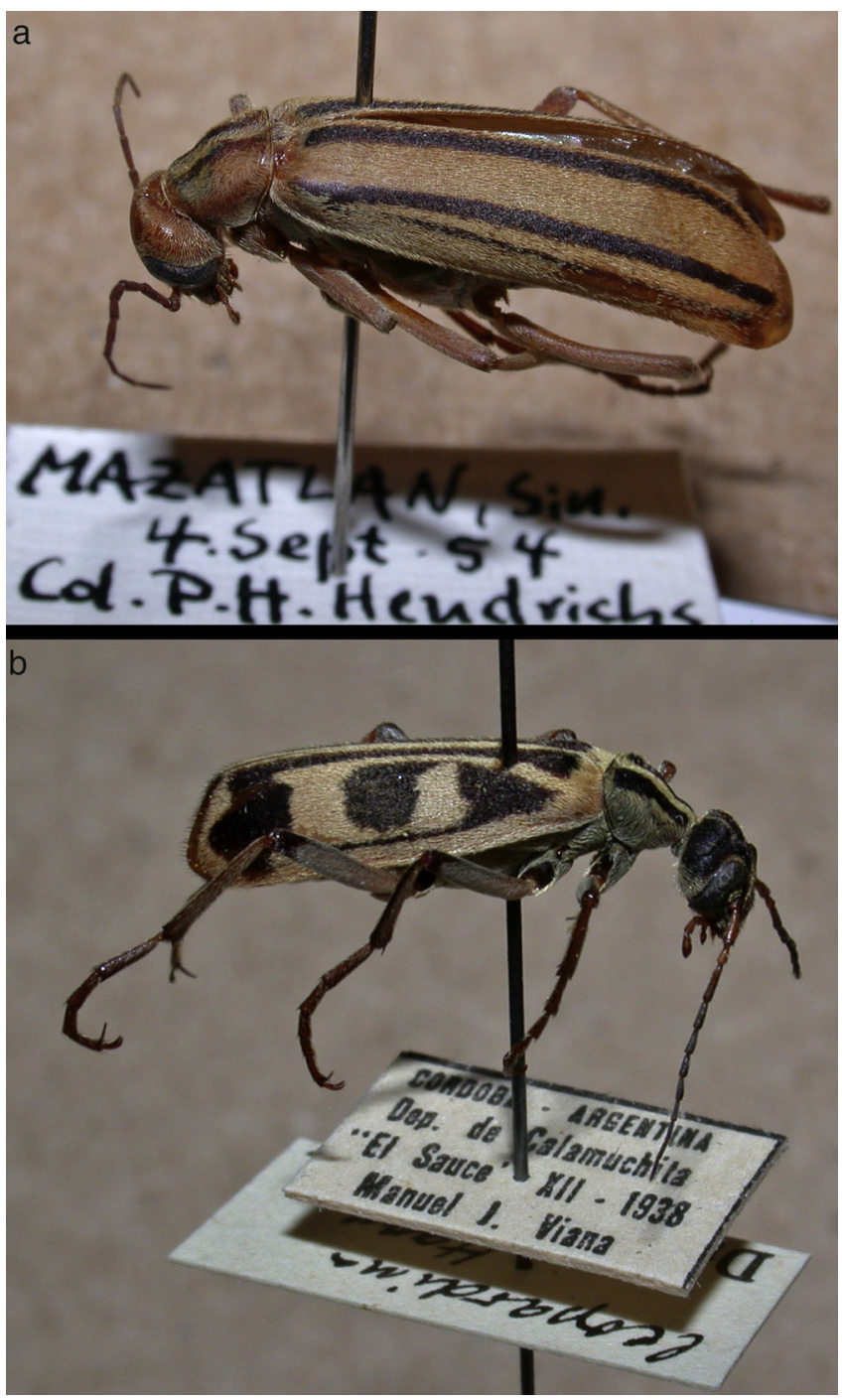

Figura 3. Ejemplares representativos de Epicauta abadona. a) Vista dorsolateral de un ejemplar de Mazatlán (Sinaloa, México) (CNIN-IBUNAM), nótese la pilosidad clara sobre algunas zonas de las bandas oscuras; y de E. leopardina. b) Vista dorsolateral de un especimen de Calamuchita (Córdoba, Argentina) (HNHM).

de la coloración tegumentaria; así, en la mayor parte del cuerpo, incluidas casi todas las zonas oscuras de los élitros, la pilosidad es de color amarillo-blanquecino; esta característica se presenta en E. abadona de la costa del Pacífico de México y EE. UU., que presenta la banda oscura exterior del élitro cubierta por pilosidad amarilla (fig. 3a). El patrón general de coloración es, además, muy distinto del de las especies del grupo estudiadas, excepto E. leopardina, ya que la banda longitudinal oscura media esta partida y ensanchada distalmente, hasta, en algunos ejemplares (paratipo), unirse con la banda exterior. Este patrón recuerda al de E. leopardina y E. nattereri, pero en estas especies la pilosidad es mucho más densa y fuerte, el patrón es distinto y la pilosidad coincide con el color del tegumento (fig. $3 b$ ).

Las afinidades filogenéticas de E. falcolarandina no pueden establecerse con el material disponible (solo 2 hembras) ya que muchos de los caracteres utilizados en las reconstrucciones filogenéticas dentro del género Epicauta son exclusivos de los machos (Pinto, 1991). Sin embargo, la presencia de láminas unguiculares superior e inferior subiguales la aleja marcadamente de E. grammica, E. subvittata, E. apure y E. aragua. El estado de conocimiento actual del grupo, sin material de referencia de algunas especies prácticamente desconocidas y la situación taxonómica incierta de otras, hacen imposible la realización de un estudio filogenético en el momento actual.

\section{Discusión}

La fauna centro y norteamericana del grupo de especies de E. vittata puede considerarse bien conocida en la actualidad (Adams y Selander, 1979; Agafitei y Selander, 1980; Pinto, 1991; Werner, 1944). Los problemas detectados se centran, en primer lugar, en el reconocimiento, análisis y alcance de una posible estructuración de la variabilidad morfológica y genética en $E$. vittata y en $E$. vitticollis, especies que presentan variaciones morfológicas geográficas acusadas y que podrían presentar niveles limitados de flujo génico entre ellas y, en segundo lugar, en la complicada diferenciación taxonómica entre $E$. dugesi y $E$. unilineata (Pinto, 1991; García-París et al., 2007). La resolución de ambos problemas, concretos y claramente diagnosticados, requiere el uso de marcadores moleculares y muestreos intensivos en las zonas de contacto entre razas geográficas o en la zona de hibridación entre especies. Por el contrario, el grado de conocimiento de las especies sudamericanas del grupo de $E$. vittata es muy distinto del de las norteamericanas. Las especies estudiadas y descritas por Fairmaire (1875; E. semivittata), Haag-Rutenberg (1880; E. leopardina y E. nattereri), Berg (1881, 1883, 1889; E. clericalis, E. missionum y E. monachica), Borchmann (1930; E. rutilifrons), Pic (1933; E. luteolineata) y Denier $(1935 ; E$. bosqi) no parecen presentar problemas taxonómicos, las descripciones son claras y detalladas, y los tipos pueden consultarse, además muchas de ellas han sido revisadas en detalle por Campos-Soldini y Roig-Juñent (2011).

Las demás descripciones plantean problemas de un tipo u otro. Las aparentemente relacionadas E. aemula y E. strigata, ambas de Brasil, constituyen un complejo muy poco estudiado, con problemas taxonómicos por resolver (Borchmann, 1917; Martínez, 1955), pero al parecer se trata de 2 especies bien diferenciadas de E. floydwerneri con la que habían estado confundidas (Haag-Rutenberg, 1880, sub E. rubriceps, non E. rubriceps Redtenbacher, 1842; Selander, 1981). Epicauta apure descrita de la cuenca del Orinoco (Adams y Selander, 1979), E. grammica descrita del Brasil (Fischer, 1827) y E. subvittata descrita de la Guayana Inglesa (Erichson, 1848), aparentemente forman un complejo de especies muy similares entre sí, cuya variabilidad podría ser continua. La descripción de E. apure (Adams y Selander, 1979), realizada sin establecer las comparaciones adecuadas con las demás especies del grupo, resulta fácilmente aplicable a E. grammica. Los propios Adams y Selander (1979, p. 256) indican que la «Description of this species is necessitated by the need for a name for use in an article on behavior and ecology now in preparation». A lo largo del texto de Adams y Selander (1979) se deslizan varias contradicciones con respecto a esta especie, ya que por ejemplo en la página 153, indican que la distribución de E. apure va desde Venezuela hasta Bolivia, 
mientras que en la descripción de la especie (página 257) limitan su distribución geográfica a la cuenca del Orinoco en Venezuela y Trinidad. Esta ambigüedad llega hasta el punto de que en la descripción original indican que $E$. apure podría ser la misma especie que E. grammica (Adams y Selander, 1979,p. 256). Se han examinado ejemplares asignables a $E$. grammica procedentes de Venezuela, Argentina, Guayana Inglesa, Ecuador, Surinam y Brasil (apéndice). Todos ellos guardan una gran semejanza entre sí, pero difieren en proporciones corporales y detalles de la coloración que no podemos evaluar adecuadamente debido al escaso número de ejemplares disponible de cada procedencia geográfica. Para complicar la situación, la descripción de Lytta subvittata Erichson, 1848, tampoco permite distinguirla de E. apure, ni de E. grammica. La resolución del problema de $E$. apure requiere una revisión taxonómica profunda, por lo que de momento consideramos oportuno mantener su estatus específico.

En definitiva, si bien la representación específica norteamericana del grupo de E. vittata presenta una taxonomía relativamente clara (con problemas concretos y bien conocidos), las especies sudamericanas adscritas a este grupo necesitan una revisión profunda de conjunto, ya que por una lado la diversidad estructural del grupo es muy grande (con E. excavata y E. semivittata muy alejadas morfológicamente del resto) y por otro, varias de las especies con amplia distribución, en particular E. grammica, podrían representar complejos de especies sin definir.

\section{Agradecimientos}

A Jesús Manzanilla por su cálida acogida e inestimable ayuda en el Instituto de Zoología Agrícola (Maracay, Venezuela); a Vilma Savini y Luis José Joly por las facilidades prestadas para la revisión de la Colección Entomológica del Museo del Instituto de Zoología Agrícola (MIZA, Maracay); a Judit Vörös y Ötto Merkl por las facilidades prestadas para el examen de material de la colección del Magyar Természettudományi Múzeum (HNHM, Budapest); a Alejandro Zaldívar y Cristina Mayorga agradecemos su amabilidad facilitándonos el acceso a las colecciones del Instituto de Biología (UNAM, Ciudad de México); a Max Barclay por permitirnos revisar la colección de coleópteros del Natural History Museum (NHM, Londres); a Weiping Xie por facilitarnos la revisión de material del Natural History Museum of Los Angeles County (NHMLA, Los Ángeles); a Marco Bologna que amablemente nos permitió examinar su colección (Roma Tre, Roma) y a Pepe Fernández (MNCN, Madrid) por su ayuda en la localización de referencias y correcciones sobre el manuscrito. La colaboración de MG-P con la Universidad Central de Venezuela ha sido posible gracias al apoyo de la (Agencia Española de Cooperación Internacional (AECI). La visita de MG-P a la colección del Magyar Természettudomány Múzeum (HNHM) en Budapest fue financiada por el proyecto Synthesys «European Commission's Research Infrastructure Action». Este estudio ha contado con el apoyo del Proyecto CGL2015-66571-P (MINECO/FEDER). Agradecemos al Instituto de Estudios Ceutíes el apoyo prestado en todo momento.
Apéndice. Material examinado. Las siglas corresponden a las siguientes instituciones: Colección Nacional de Insectos del Instituto de Biología de la Universidad Nacional Autónoma de México-CNIN-IBUNAM(Ciudad de México); Estación de Biología de Chamela (EBCH) del Instituto de Biología de la Universidad Nacional Autónoma de México (Jalisco, México); El Colegio de la Frontera Sur -ECOSUR- (San Cristóbal de las Casas, México); Hungarian Natural History Museum (HNHM), Magyar Természettudományi Múzeum (Budapest, Hungría); Colección M. A. Bologna (MAB), Universita Roma Tre (Roma, Italia); Museo del Instituto de Zoología Agrícola (MIZA) de la Universidad Central de Venezuela (Maracay, Venezuela); Natural History Museum -NHM- (Londres, UK); Natural History Museum of Los Angeles County -NHMLA- (California, Estados Unidos).

\section{Epicauta (Epicauta) abadona Skinner, 1904}

MÉxico: SinAloA: Mazatlán: 17 al 20-X-73, 1, luz mercurial al mar (J. Hendrichs, S) (Ex Colección Jorge Hendrichs S.) (CNIN-IBUNAM); Mazatlán: 4-IX-1954, 3 (P.H. Hendrichs, J. Hendrichs) (Ex Colección Jorge Hendrichs S.) (CNINIBUNAM). SonOrA: Álamos, 390 m: 26/27-VII-1995, 2, luz negra (F.A. Noguera leg.) (EBCH). EE. UU.: ArIzONA: Phoenix: 8-VII-51, 1, at light (coll Y. Sedman) (Epicauta abadona Skinner det. R.B. Selander 1953) (HNHM); Cochise Co.: $17 \mathrm{mi}$. E Douglas: 3-VIII-1967, at light (L.D. Anderson leg.) $(n=1)$ (M.A. Bologna det., 1977) (MAB); Huachuca Mountains, 78000 ft.: 18.VI/15-IX-1899 (R.E. Kunzé) (Hope Coll. 1917-30) (Epicauta abadona Skinner, ex descr. K.G. Blair det.) (NHM); Patagonia Mountain: 20-VIII-1949, 1, hembra (F.H. Parker leg.) (8020 F.H. Parker Collection) (E. abadona, F. Werner det.) (MIZA); Phoenix: 5-IX-1947, 1 hembra (Parker) (NHM).

Epicauta (Epicauta) apure Adams et Selander, 1979

Trinidad \& Tobago: Trinidad: at light Trinidad St. Agustine 1-ix-1940// E.C. Humphries BM 1954-636// Epicauta grammica Fischer det. Kaszab (HNHM). Venezuela: Zulia: El Tucuco: 5-I-1983, 1, primary forest (T. Racheli leg.)/ Epicauta (Epicauta) apure Selander; M.A. Bologna det., 1996 (MAB).

Epicauta (Epicauta) aragua Adams et Selander, 1979

Colombia: Bolívar SFF: Los Colorados, Villa Roca, 180 m, 9 $9^{\circ} 4^{\prime} \mathrm{N}-75^{\circ} 07 » \mathrm{~W}:$ 6/24-I-2001, 1, malaise (E. Deulufeut leg.) (M 2421)/ Epicauta (Epicauta) aragua Selander; M.A. Bologna det., 2002 (MAB). MagdalenA: Aracataca 1912 II// Ujhelyi// Epicauta grammica Fischer. det. Kaszab (HNHM). Slg. R. Oberthür Eing. Nr. 4-1956, 3. Costa RicA: GuANACASTE: Santa Rosa National Park: 6-XII-1979, 1 (D. H. Janzen) (CNIN-IBUNAM). MÉxICO: (sin datos de localidad adicionales) Slg. R. Oberthür Eing. Nr. 4-1956// Epicauta grammica Fischer det. Kaszab (HNHM) (error de etiquetado). PANAMÁ: Canal Zone, XII-1960, G. Frey// Epicauta grammica Fischer. det. Kaszab (HNHM). Venezuela: Anzoátegui: Barcelona: Puerto La Cruz// H kulzer le. 1959// Epicauta grammica Fischer. det. Kaszab (HNHM) (Epicauta aragua). AraguA: Cagua, 450 m, 28-V-58// Col. A. Fernández// Epicauta grammica Fischer. det. Kaszab, 2 (HNHM); El Limón, 450 m 5-IV-61// C.J. Rosales 
leg.// Epicauta grammica Fischer. det. Kaszab (HNHM); Maracay P. Vogl. Jul 34// Epicauta grammica Fischer det. Kaszab, 5 (HNHM); Maracay, 450 m 2-IV-63// en luz// C.J. Rosales leg.// Epicauta grammica Fischer. det. Kaszab (HNHM); Rancho Grande, Maracay, XI.1960 G. Frey// Epicauta grammica Fischer. det. Kaszab (HNHM). CARAвoвo: San Diego: 17-V61// N. Ángeles leg.// Epicauta grammica Fischer. det. Kaszab (HNHM). Monagas: $42 \mathrm{~km}$ SE Maturin: 5-VI-1958, 1 (A. Menke); 20-VI-1958, 3 (A. Menke); 8-VII-1958, 1 (A. Menke)/ Epicauta grammica, J. D. Pinto det., 1988 (NHMLA); $60 \mathrm{~km} \mathrm{SE}$ Maturin: 23-VI-1958, 1 (A. Menke); 24-VI-1958, 2 (A. Menke); 29-VI-1958, 1 (A. Menke); 1-VII-1958, 1 (A. Menke)/ Epicauta grammica, J. D. Pinto det., 1988 (NHMLA).

\section{Epicauta (Epicauta) dugesi Werner, 1957}

MÉxico: Guerrero: Rodecia: 25-V-86, 1 (E. Barrera, H. Brailovsky) (CNIN-IBUNAM); Zihuatanejo: 2-XI-64, 1 (J. Hendrichs S.) (CNIN-IBUNAM). NAYARIT: P.H. Aguamilpa, San Rafael, Arroyo de la Virgen: 1-XI-1991, 1 (R. Barba, E. Barrera) (CNIN-IBUNAM). OAXACA: Km. 14 de la carretera Pinotepa - Puerto Escondido: 3-IX-90, 1 (E. Barrera, A. Cadena) (CNIN-IBUNAM); Val de Flores: 21-V-61, 1 (L. Vázquez) (CNIN-IBUNAM). SAn LuIs Potosí: Colección E. Dugès (impreso) Huasteca de San Luis Pot. 758 (manuscrito tinta negra) D-2991 (manuscrito en tinta roja)/ Epicauta lemniscata vittata/ Holotipo Epicauta vittata Dugès (etiqueta impresa en rojo) (CNIN-IBUNAM) (este no es un tipo ya que Dugès nunca llegó a describir esta especie, ver García-París et al., 2007). SinALOA: Mazatlán: 18/20-VII-1956, 1 (A. E. Lewis) (NHMLA). SonORA: Cocorit: 11-VI-1961, 2 (Menke; Stange)/ Epicauta tamara, R. B. Selander det. (NHMLA). VerAcruZ: Tuxtla: 26V-72, 1 (H. Brailovsky) (CNIN-IBUNAM).

Epicauta (Epicauta) excavata (Klug, 1825)

Argentina: Misiones: Santa Anna Dr. Cernostitov// Epicauta excavata Klug det. Kaszab (HNHM). SALtA: La Viña: II-1984, 1 (D. Carpintero leg.) (MAB); Rosario de Lerma: II-1984, 1 (D. Carpintero leg.)/ Epicauta (Epicauta) excavata Klug; M.A. Bologna det., 2002 (MAB). BRAsIL: Epicauta excavata Klug det. Dr. Z. Kaszab (HNHM). Nova Teutonia: S. Catar. (Santa Catarina)// XII-1955 Plaumann// Epicauta excavata Klug det. Dr. Z. Kaszab, 3 (HNHM); Santa Catarina Plaumann 25.11.50// Epicauta excavata Klug det. Dr. Z. Kaszab, 3 (HNHM); Santa Catarina: I-1971, 1 (J. Plaumann leg.) (Epicauta excavata Klug, J.D. Pinto det., 1987) (MAB). Río d J Brasil Jan. 1947 Coll. Wygodzinski// Epicauta excavata Klug det. Kaszab (HNHM). Río Grande Do Sul: Santo Augusto: I-1969 (O. Roppa leg.) ( $\mathrm{n}=1)$ (Epicauta (Epicauta) sp. nov. aff. caustica M.A. Bologna det.) (MAB). SÃo PAulo: San José dos Campos: 1/4-XI-1960, 1 (D. Tiemann); 6/9-XI-1960, 1 (D. Tiemann); 10/13-XI-1960, 1 (D. Tiemann); 22-I-1963, 1 (D. Tiemann)/ Epicauta excavata Klug, R. B. Selander det., 1975 (NHMLA). Sabara - Bello Horizonte. Rio das Velhao. A.G.N. Chalmers B.M.1932-11, 2 (NHM). PARaguay:Villa Rica Colonia Sudética// Epicauta excavata Klug det. Dr. Z. Kaszab (HNHM).

\section{Epicauta (Epicauta) grammica (Fischer, 1827)}

Argentina: Chaco Col. Ohnheiser// Epicauta grammica Fischer. det. Kaszab (HNHM). CoRrIENTES: $25 \mathrm{~km}$ al $\mathrm{N}$ de
Loreto: 15-VI-2000, 1 (G. Carpaneto leg.) (MAB); Laguna Ibera, Colonia Pellegrini, Reserve Station: 24-XII-2000, 5, light trap (L. Facchinelli leg.) (MAB); Laguna Ibera, Colonia Pellegrini: 1/12-XI-1999, 2(G. Carpaneto leg.) (MAB). Hungarian Soil-Zool Exp. Argentina. Prov. Santa Fe. Coronda. 22-III-65// Nr. P-B 309 leg. Mahunka// Epicauta grammica Fischer det. Kaszab (HNHM). Misiones. 1932. K.J. Hayward B.M. 1933187// (NHM); 8.II.950 leg. J. Förster// Epicauta grammica Fischer. det. Kaszab (HNHM). Saenz Peña. Chaco. K.J. Hayward B.M. 1933-187, 2 (NHM). Bolivia: Río Yacuma Espíritu

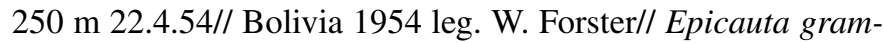
mica Fischer. det. Kaszab (HNHM). BRAsIL: Brazil// grammica Fisch t. Haag// F. Bates 81-19, 3 (NHM). Brazil: Est. Pará: Santarem, Lt. trap, 27-April 1963 F.G. Werner// E. grammica F.G. Werner Collection// Epicauta grammica (Fisch.) det. F. Werner, 2 (NHM). Guayaba Brasilien// Epicauta grammica Fischer det. Kaszab (HNHM). Río d J (de Janeiro) Brasil Rez. 1943 Coll. Wygodzinsky// Epicauta grammica Fischer. det. Kaszab, 2 (HNHM). SAnta CATARINA: Nova Teutonia: 23-XI-1951, 2 (F. Plaumann leg.) (E. grammica F.G. Werner Collection) (E. grammica, F. Werner det.) (MIZA); Nova Teutonia: F. Plaumann leg. 14.11.04// Epicauta grammica Fischer det. Kaszab (HNHM). São Paulo: San José dos Campos: 6/9-IX-1960, 1 (D. Tiemann); 6/8-X-1960, 1 (D. Tiemann);10/13-X-1960, 1 (D. Tiemann);14/17-X-1960, 1 (D. Tiemann); 24/25-X-1960, 1 (D. Tiemann); 1/4-XI-1960, 1 (D. Tiemann);/ Epicauta grammica, J. D. Pinto det., 1988 (NHMLA). Unt. Amaz. Taperinha b. Santarem 21-31.VII-'27 Zerny// Epicauta grammica Fischer. det. Kaszab, 4 (HNHM). EcuAdor: NAPO: Coca: VI-1983, 1 (G. Onore leg.) (MAB); XI-1987, 1 (G. Onore leg.) (MAB). PARAgUAY: Assomption 1936// Epicauta grammica Fischer. det. Kaszab (HNHM). Fiebrig Paraguay S. Bernardino// Epicauta grammica Fischer. det. Kaszab, 3 (HNHM). Hungarian SoilZool Exp. Paraguay. Puerto P. Stroessner. 26-29-III-65// Nr. P 11 leg. Mahunka \& Zicsi// Epicauta grammica Fischer. det. Kaszab (HNHM). Puerto Midina 1936// Epicauta grammica Fischer. det. Kaszab (HNHM). Puerto Stroessner C. Dlouhy leg. VIII.77// Epicauta grammica Fischer. det. Kaszab (HNHM).

Epicauta (Epicauta) leopardina (Haag-Rutenberg, 1880)

Argentina: 1 (García leg.)/ Epicauta (Epicauta) leopardina Haag-R.; M.A. Bologna det., 1979 (MAB). Córdoba, Dep. de Calamuchita «El Sauce» XII-1938 Manuel J. Viana// Epicauta leopardina Klug det. Dr. Z. Kaszab, 2 (HNHM). Hungarian Soil-Zool. Expedition, Prov. Santa Fe, between Santa Fe and Reconquista, 23-XII-1965// Nr. P-B 311 leg. Mahunka// Epicauta leopardina Klug det. Dr. Z. Kaszab (HNHM). Metan (Salta) I-1906// Vezènyi// Epicauta leopardina Klug det. Dr. Z. Kaszab, 4 (HNHM). Tucumán// Epicauta leopardina Klug det. Dr. Z. Kaszab, 3 (HNHM); Tucumán 1906// Vezènyi// Epicauta leopardina Klug det. Dr. Z. Kaszab, 3 (HNHM); Tucumán I-1906// Vezènyi// Epicauta leopardina Klug det. Dr. Z. Kaszab (HNHM); Río Salí: 7-XII-1898, 1 (Pablo?) (NHM). Villa de Soto: 8-II-1992, 2, limpiando Modul Tiles (M. Pinzano leg.) (MAB). MÉXICO: CAMPECHE: Pital, La Encarnación: 6 (NHMLA) (error de etiquetado). PARAgUAY: Gran Chaco Filadelfia I-1985 leg. Schuster// Epicauta leopardina Klug det. Dr. Z. Kaszab (HNHM) 


\section{Epicauta (Epicauta) luteolineata Pic, 1933}

Argentina: Wagner Brothers '04// La Palisa bei Bracho Bordo du Rio Salado// Chaco de Santigo del Estero Argentina// Epicauta luteolineata Pic cum typo comp. Dr. Z. Kaszab 1963, 3 (HNHM). Río Cuarto// Epicauta luteolineata Dr. Z. Kaszab det. (HNHM). Capilla d. Monte Cordoba, Coll. Prof Hosseus// La Palisa bei Bracho Bordo du Rio Salado// Chaco de Santigo del Estero Argentina// Epicauta brevebasalis Pic cum typo comp. Dr. Z. Kaszab 1963 (HNHM).

Epicauta (Epicauta) missionum (Berg, 1881)

Paraguay: Assomption 1936// Epicauta missionum Berg det. Dr. Z. Kaszab (HNHM).

\section{Epicauta (Epicauta) monachica (Berg, 1883)}

Argentina: Argentina Galleros (hand written)// Paratypus Epicauta monachica Berg, 1883, 2 (HNHM). Cruz de Leche, H. Isler// Epicauta monachica Berg det. Kaszab (HNHM). Mendoza// Epicauta monachica Berg det. Kaszab (HNHM). Salta, San Carlos// 22-I-1950 Monrós y Willinek// Epicauta monachica Berg det. Dr. Z. Kaszab, 4 (HNHM); San Carlos, Salta// 22-I-1950 Monrós y Willinek// Epicauta monachica Berg det. Kaszab, 5 (HNHM). SAN LuIs: San Gerónimo: II-1974, 1 (M. Viana leg.)/ Epicauta (Epicauta) monachica Berg; T. Barriga det. (MAB). Tucumán// 1941 Hayward// Epicauta monachica Berg det. Dr. Z. Kaszab, 4 (HNHM); Tucumán, 8-X-1948, Coll. Wygodzinski// Epicauta monachica Berg det. Kaszab (HNHM); Tucumán, XI.49, De la Sota leg.// Epicauta monachica Berg det. Dr. Z. Kaszab, 5 (HNHM).

\section{Epicauta (Epicauta) occidentalis Werner, 1944}

EE. UU.: Alabama: Tuscaloosa: 7-VII-1952, 1 (B.D. Valentine leg.) (E. occidentalis F.G. Werner Collection) (E. occcidentalis, F. Werner det.) (MIZA). LouisianA: Harahan, VIII-25-44 F.G. Werner// Epicauta occidentalis Werner. Werner det. 54, 2 (HNHM). MissouRI: Hattiesburg: 12-VII-1952, 1, at (C.B. Segars leg.) (E. occidentalis F.G. Werner Collection) (E. occcidentalis, F. Werner det.) (MIZA). NEBRASKA: Lincoln: 19-VII-1917, 1 (R.W. Dawson) (BM 1993-53) (Epicauta occidentalis, F.G. Werner collection) (NHM). TennesEE: Ben. Co.: Camden: 5-VII-1953, 1 (R. X. Schick) (NHMLA). TEXAs: Bexar Co.: San Antonio: 13-IX-1972 (D. Anderson leg.) $(n=1)$ (M.A. Bologna det., 1977) (MAB).

\section{Epicauta (Epicauta) semivittata (Fairmaire, 1875)}

CHILE: Chili Reed (partly hand written)// Cantharis semivittata Fairm. type (hand written)// semivittata Fairm. type (hand written)// hemigramma Mäkl. t. Haag// hemigramma Mäkl.// (NHM).

\section{Epicauta (Epicauta) temexa Adams et Selander, 1979}

MÉxico: SAn LuIs Potosí: Micos - Ciudad Valles: 2-IX-78, col. diurna, 1 (G. Figueroa)/ Epicauta temexa Adams y Selander. J. D. Pinto det., 1987/8 (CNIN-IBUNAM); San Luis Potosí: 1 (L. Ancona) (CNIN-IBUNAM). TAMAUliPAS: La Pesca: 8-X1985, 2 (F. Arias, M. García, L. Cervantes) (CNIN-IBUNAM); Río La Palas (?): 24-X-1985, 1 (A. Gueno) (CNIN-IBUNAM); Villa Aldama: 8-VI-1979, 1 (R. Valencia) (CNIN-IBUNAM). VERACRUZ: $1.3 \mathrm{~km}$ al S de Álamo: 19-X-1996, 1 (S. Zaragoza) (CNIN-IBUNAM); El Higo: 25-VI-76, col. diurna, 4 (G. Figueroa)/ Epicauta temexa Adams y Selander. J. D. Pinto det., 1987/8 (CNIN-IBUNAM).

\section{Epicauta (Epicauta) unilineata Champion, 1892}

Guatemala: (Sintipo) San Gerónimo, Guatemala. Champion/ Sp. figured/ Godman - Salvin Coll., Biol. Centr.-Amer./ Epicauta unilineata $\mathrm{Ch}(\mathrm{n}=1)$ (NHM). Paratype (círculo con borde amarillo; impresa)/ Guatemala, 5000/ Sp. figured/ Godman - Salvin Coll., Biol. Centr.-Amer./ Epicauta unilineata $\mathrm{Ch}$ $(\mathrm{n}=4)(\mathrm{NHM})$. Syntype (círculo con borde azul claro; impresa)/ Guatemala, 5000/ Sp. figured/ Godman - Salvin Coll., Biol. Centr.-Amer./ Epicauta unilineata $\mathrm{Ch}(\mathrm{n}=4)(\mathrm{NHM})$. Type (círculo con borde rojo; impresa)/ Guatemala, 5000/ Sp. figured/ Godman - Salvin Coll., Biol. Centr.-Amer./ Epicauta unilineata Ch (NHM). México: OAxaCA: Camino a Mixtequilla, Tehuantepec: 22-IX-55, 10 (CNIN-IBUNAM); Tehuantepec: 20-IX-55, 3 (CNIN-IBUNAM); Tehuantepec: 2-V-81, 1 (J. García) (CNINIBUNAM). NiCARAgUA: GRANADA: Domitila Wildlife Reserve, tropical dry forest $11^{\circ} 57^{\prime} \mathrm{N}-85^{\circ} 46^{\prime} \mathrm{W} / /$ at light 9/14-VI-2007 leg. O. Merkl, N. Bálint y T. Németh, 4 (HNHM), 1 (MNCN). RIVAS: vic. Rancho Santana: 27-V-2003, 2 (Van Den Berghe leg.)/ Epicauta (Epicauta) unilineata (Champ.); M.A. Bologna det., 2005 (MAB).

\section{Epicauta (Epicauta) vittata (Fabricius, 1775)}

EE. UU.: ILlinoIS: La Salle Co.: 30-VII-1937, 1 (F.G. Werner leg.) (F.G. Werner Collection) (E. vittata, F. Werner det. 47') (MIZA); Otawa: 16-VIII-1947, 1 (F.G. Werner leg.) (E. vittata F.G. Werner Collection) (E. vittata, F. Werner det.) (MIZA); Otawa: 16-VIII-1947, 1, food plant Solanum tuberosum (8018) (F.G. Werner collection) (Epicauta vittata (F.), F. Werner det.) (NHM). Tennesee: Putman Co.: Camden: 1 (S. Odmundson) (NHMLA).

\section{Epicauta (Epicauta) vitticollis (Haag-Rutenberg, 1880)}

Guatemala: Alta Verapaz: Coban: 3-I-1973, 2 (T. W. Taylor) (NHMLA). EL PETÉN: San Luis Las Cañas: 26-I-1973, 1 (T. W. Taylor) (NHMLA). Guatemala: 1931, 2 (Giaquinto leg.)/ Epicauta (Epicauta) vitticollis Haag-R.; Z. Kaszab det., 1975 (MAB). Petén: Santa Elena: 22/23-VIII-1989, 3 (MAB). Type (círculo con borde rojo, impresa)/ Guatimal (manuscrita, ilegible)/ F. Bates 81-19 (impresa)/ vitticollis typ Haag (manuscrita) (NHM). México: CAMPECHE: $30 \mathrm{~km}$ al $\mathrm{S}$ de Xpujil: 20-III-1982, 2 (M. García) (CNIN-IBUNAM); Municipio de Calakmul: Calakmul: 17-XI-2001, 1 (H. Brailovsky, E. Barrera, L. Cervantes) (CNIN-IBUNAM); Reserva de Calakmul: Xpuhil: 15-XI-2001, 1 (H. Brailovsky, E. Barrera, L. Cervantes) (CNIN-IBUNAM). Chiapas: Agua Azul: 1-V-1974, 2, col. nocturna (E. Barrera)/ Epicauta vitticollis Haag, J. D. Pinto det., 1987 (CNIN-IBUNAM); Chajul: 14/17-IV-1993, 2, luz negra (R. Ayala y F.A. Noguera leg.) (EBCH); Corozal: 25-V-1984, 1 (A. Ibarra) (CNIN-IBUNAM); Crucero Reserva, $16^{\circ} 47^{\prime} 509 » \mathrm{~N}-$ 9055'006»O: 13-VII-2004, 1 (A. Ibarra) (CNIN-IBUNAM); La Canja, Chansaya: VII-1977, 1, a la luz (P. Reyes C.)/ Epicauta vitticollis Haag, J. D. Pinto det., 1987 (CNIN-IBUNAM); Mal Paso: La Selva: 1 al 2-VI-1974, 1 (C. ARR) (CNIN-IBUNAM); Municipio Ocozocuautla: Reserva El Ocote: $2 \mathrm{~km}$ al SO de Cuauhtémoc: 7-X-1979, 1 (O. Gómez N. leg.) (ECOSUR); Municipio Tuxtla Gutiérrez: Mal Paso: 2-VI-1974, 1 (Col. A.R.R. CIES) (891) (ECOSUR); Ocosingo, Chajul, Reserva Montes Azules: 28-IV al 5-V-1986, 5 (F. Arias, R. Barba, L. Cervantes) (CNIN-IBUNAM); Ocosingo, Montes Azules: 9 al 
14-VII-1987, 1 (R. Barba, L. Cervantes) (CNIN-IBUNAM); Ocosingo: 15-VII-1987, 1 (L. Cervantes) (CNIN-IBUNAM); Palenque, 600 m: 17/23-VI-1994, 1 (V. Maly leg.) (94/A/09, M38) (MAB); Palenque, Chacamax: 25-VI-1989, 1 (A. Cadena, L. Cervantes) (CNIN-IBUNAM); Palenque: 22-VI-1979, 1 (E. Barrera)/ Epicauta vitticollis Haag, J. D. Pinto det., 1987 (CNIN-IBUNAM); Reserva El Ocote: 30-V-1993, 1 (E. Barrera) (CNIN-IBUNAM). Mexico Tarnier// Paratypus 1880 Lytta vitticollis Haag-Rutenberg (HNHM). OAXACA: Presa Temascal: 11-III-78, 1, col. diurna (E. Mariño) (CNIN-IBUNAM); Uxpanapa: 24-VII-76, 3, col. nocturna (Barrera) (CNIN-IBUNAM). Quintana Roo: Felipe Carrillo, Puerto Buenavista: 22-VI1989, 4 (A. Cadena, L. Cervantes) (CNIN-IBUNAM); Km 146 carretera Chetumal - Puerto Juárez: 17-III-1982, 1 (M. García) (CNIN-IBUNAM); X-Can: 20-VI-1963, 1 (T. W. Taylor); 20-VI-1967, 1 (E. Welling) (NHMLA). TABASCO: Frontera: 1 (C.C. Hoffmann) (CNIN-IBUNAM); Teapa: 1 (Ant. Col. Mus. de Hist. Nat., 758) (CNIN-IBUNAM). VeraCrUZ: $7.2 \mathrm{~km} \mathrm{NE}$ Sontecomapan: 31-X-1989, 1, col. nocturna (S. Zaragoza, A. Juárez) (CNIN-IBUNAM); Cosoleacaque: 18-III-2003, 1 (L. Rivera) (CNIN-IBUNAM); Estación Biológica Los Tuxtlas, 160 msnm: 21-IV-1985, 1 (A. Ibarra); 30-IV-1985, 1 (A. Ibarra); 23-V-1985, 1 (A. Ibarra); 19-VI-1985, 1 (A. Ibarra); 24-VII1985, 1 (E. Ramírez); 24-VII-1985, 1 (P. Sinaca); 27-VII-1985, 1 (E. Ramírez) (TUXTLAS-IBUNAM); Estación Biológica Los Tuxtlas, 170 msnm: 21-V-1985, 1 (E. Ramírez) (TUXTLASIBUNAM); Estación Biológica Los Tuxtlas: 15-III-1975, 1; 9-V-1975, 1, col. nocturna; 14 al 16-XII-1975, 1; 18-I-1980, 2 (E. Barrera); 18-I-1980, 1 (H. Brailovsky); 20 al 26-II-1985, 1 (A. Ibarra); 21-II-1985, 1 (H. Velasco); 30-II-1985, 10 (A. Ibarra); 3-III-1985, 1 (A. Ibarra); 6-III-1985, 1 (A. Ibarra); 28III-1985, 2 (A. Ibarra); 5-IV-1985, 1 (A. Ibarra); 23-IV-1985, 1 (P. Sinaca, A. Ibarra); 24-IV-1985, 1 (C. Mayorga);13-V1985, 2 (P. Sinaca); 14-V-1985, 1 (L. Cervantes); 20-V-1985, 1 (A. Ibarra); 18-VI-1985, 1 (A. Ibarra); 14-IX-1985, 2 (F. Arias); 11-XI-1985, 1 (A. Ibarra, P. Sinaca, E. Ramírez); 18IV-1989, 8 (E. Barrera, J.L. Colin); 21-IV-1989, 1 (J. L. Colin); 28-VI-1989, 1 (M. A. Pérez); 4-IX-1989, 1 (J.L. Colin, H. Rojas); 29-VI-1989, 1 (M. A. Pérez); 30-VI-1989, 1 (M. A. Pérez); 3 al 6-VII-1989, 1 (A. Villegas); 20-IX-1989, 1 (J.L. Colin, H. Rojas); 17-IV-1990, 3 (S. Zaragoza); 19-IV-1990, 1 (S. Zaragoza); 26-IV-1991, 1 (G. Ortega, C. Mayorga) (CNINIBUNAM); Estación Biológica Los Tuxtlas: camino a Laguna Escondida: 9-IV-1990, 1 (G. Ortega, C. Mayorga); 24-III1991, 1 (G. Ortega, C. Mayorga) (CNIN-IBUNAM); Estación Biológica Los Tuxtlas: camino a Sontecomapan: 13-X-1989, 1 (J.L. Colin, H. Rojas) (CNIN-IBUNAM); La Cabaña (?): 11-I-1972, 1 (CNIN-IBUNAM); Las Chispas: 15-IX-1977, 1, col. diurna (H. Brailovsky) (CNIN-IBUNAM); Las Choapas: 28-V-1976, 1 (C. Mariño)/ Epicauta vitticollis Haag, J. D. Pinto det., 1987 (CNIN-IBUNAM); Los Tuxtlas, Las Cabañas: 11-I-1972, 1 (H. Brailovsky) (CNIN-IBUNAM); Los Tuxtlas: Camino a Playa Escondida: 2-XI-1989, 2 (S. Zaragoza) (CNIN-IBUNAM); Los Tuxtlas: II-63, 1 (CNIN-IBUNAM); Motzorongo: IX-1937, 1 (P. Roveglia) (CNIN-IBUNAM); Playa Escondida: 27-III-1976, 2, col. nocturna (E. Barrera) (CNINIBUNAM); Río Playa Escondida: 22-II-1985, 1 (F. Arias);
22-II-1985, 3 (H. Velazco) (CNIN-IBUNAM); San Andrés Tuxtla: 18 al 20-I-1980, 1 (H. Brailovsky, E. Barrera); 2-III-1990, 1 (J. Razowski) (CNIN-IBUNAM); San Andrés Tuxtla: La Palma: 9-XI-1989, 1 (J. L. Colin, H. Rojas) (CNIN-IBUNAM); San Andrés Tuxtla: Laguna Encantada: 18-IV-1984, 3 (E. Barrera, J. L. Colin) (CNIN-IBUNAM); Santiago Tuxtla: C. del Vigía: 2-IX-1967, 1 (S. Zaragoza)/ Epicauta vitticollis Haag, S. Zaragoza det. (CNIN-IBUNAM). YUCATÁN: Chichenitzá,

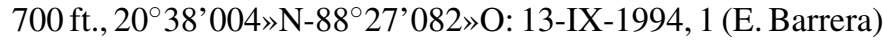
(CNIN-IBUNAM).

\section{Referencias}

Adams, C. L. y Selander, R. B. (1979). The biology of blister beetles of the vittata group of the genus Epicauta (Coleoptera, Meloidae). Bulletin of the American Museum of Natural History, 162, 139-266.

Agafitei, N. J. y Selander, R. B. (1980). The first instar larvae of the vittata group of the genus Epicauta (Coleoptera: Meloidae). Journal of the Kansas Entomological Society, 53, 1-26.

Berg, F. G. C. (1881). Revision der argentinischen Arten der Gattung Cantharis Entomologische Zeitung herausgegeben von dem entomologischen Vereine zu Stettin, 42, 301-309.

Berg, F. G. C. (1883). Doce heterómeros nuevos de la fauna argentina. Anales de la Sociedad Científica Argentina, 15, 66-78.

Berg, F. G. C. (1889). Quadraginata Coleoptera nova Argentina. Anales de la Universidad de Buenos Aires, 4, 105-157.

Borchmann, F. (1917). Pars 69. Meloidae, Cephaloidae. En E. Schenkling (Ed.), Coleopterorum Catalogus auspiciis et auxilio (p. 208). Berlin: W. Junk.

Borchmann, F. (1930). Alleculidae y Meloidae descriptos por Fr. Borchmann (Hamburgo). Revista de la Sociedad Entomológica Argentina, 3 85-100.

Campos-Soldini, M. P. (2011). A new species group of the genus Epicauta Dejean of Southern South America, the bella Group (Coleoptera: Meloidae) Neotropical Entomology, 40, 575-586.

Campos-Soldini, M. P. y Roig-Juñent, S. A. (2011). Redefinition of the vittata species group of Epicauta Dejean (1834) (Coleoptera: Meloidae) and taxonomic revision of the species from southern South America. Zootaxa, 2824 $21-43$

Campos-Soldini, M. P. y Roig-Juñent, S. A. (2015). Phylogenetic analysis and redefinition of the maculata species group of Epicauta (Meloidae: Meloinae: Epicautini). Insect Systematics and Evolution, 46431-46470.

Champion, G. C. (1892). Family Meloidae. En: F. E. Godman y O. Salvin (Eds.) Biologia Centrali-Americana. Coleoptera Heteromera, Vol. 4. (pp. 369-448, láms.: 17-21). Londres: Dulau \& Co.

Denier, P. (1935). Coleopterorum americanorum familiae meloidarum. Enumeratio synonymica. Revista de la Sociedad Entomológica Argentina, 7, $139-176$.

Dohrn, C. A. (1876). Anzeichnungen über einige Coleoptera Cordovana (Argentina). Stettiner Entomologischen Zeitung, 37, 405-412.

Erichson, W. F. (1848). Die Insekten in Schomburgk's Reisen in Britisch-Guiana. Schomburgk's Reisen in Britisch-Guiana, Leipzig, 3, 570-574.

Fabricius, J. C. (1775). Systema entomologiae. Sistens Insectorum classes, ordines, genera, species etc. Korte: Flesburgi et Lipsiae.

Fabricius, J. C. (1801). Systema Eleutheratorum secundum ordines, genera, species: adiectis synonymis, locis, observationibus, descriptionibus, Vol. 2 Kiel: Impensis Bibliopolii Academici Novi, Kiliae.

Fairmaire, L. (1875). Révision des Hétéroméres du Chili. Bulletin de la Société Entomologique de France, 5éme série, 5, 191-200.

Fischer, J. B. (1827). Tentamen conspectus Cantharidiarum. Disertatio inauguralis, quam pro summis in Medicina et Chirurgia honoribus legitime obtinendis eruditorum examini subjicit. Lindauer: Monachii.

García-París, M., Buckle, D. y Parra-Olea, G. (2007). Catálogo taxonómicogeográfico de los coleópteros de la familia Meloidae de México. Graellsia, $63,165-258$ 
García-París, M. y Ruiz, J. L. (2013). Description of a new species of Epicauta (Coleoptera: Meloidae) from Mexico and comments on the Epicauta cupraeola species-group. Graellsia, 69, 57-77.

Gyllenhal, L. (1817). Appendix. Descriptiones novarum specierum insectorum. ad C.J. Schöenherr Synonymia insectorum oder versuch einer synonymie, 1(3), 1-266. Leverentziana, Scaris.

Haag-Rutenberg, J. G. (1880). Beiträge zur Kentniss der Canthariden. Deutsche Entomologische Zeitschrift, 24, 17-90.

Klug, J. C. F. (1825). Entomologiae Brasilianae specimen alterum, sistens insectorum coleopterorum nondum descriptorum centuriam. Nova Acta Physico-Medica Academiae Caesareae-Leopoldino-Carolinae. Naturae Curiosorum, 12, 421-476.

LeConte, J. L. (1862). Classification of the Coleoptera of North America. Prepared for the Smithsonian Institution. Part I. Smithsonian Miscellaneous Collections, 136, 1-286.

Lemus-Jiménez, L. J. y Ramírez, N. (2002). Fenología reproductiva en 3 tipos de vegetación de la planicie costera de la península de Paraguaná, Venezuela. Acta Científica Venezolana, 53, 266-278.

Mäklin, F. W. (1875). Neue Canthariden. Acta Societatis Scientiarum Fennicae, 10, 597-632.

Martínez, A. (1955). Notas sobre Meloidae IV. Una nueva especie y un nuevo nombre de Epicautas bolivianas (Col. Meloidae). Neotropica, 1, 55-58.

Mijares-Urrutia, A. y Arends, R. A. (2000). Herpetofauna of Estado Falcon northwestern Venezuela: a checklist with geographical and ecological data. Smithsonian Herpetological Information Service, 123, 30.

Mijares-Urrutia, A. y Arends, A. (2001). A new toad of the Bufo margaritifer complex (Amphibia: Bufonidae) from northwestern Venezuela. Herpetologica, 57, 523-531.
Pic, M. (1933). Nouveautés diverses. Mélanges Exotico-Entomologiques, 61, 3-36.

Pinto, J. D. (1991). The taxonomy of North American Epicauta (Coleoptera: Meloidae), with a revision of the nominate subgenus and a survey of courtship behavior California: University of California Publications in Entomology, 110.

Pinto, J. D. y Bologna, M. A. (1999). The New World genera of Meloidae (Coleoptera): a key and synopsis. Journal of Natural History, 33, 569-620.

Rojas, M. A. (1857). Description d'une nouvelle espece de Coléoptéres de la repúblique de Venezuela. Revue et Magasine de Zoologie, 9, 441-444.

Say, T. (1824). Descriptions of the coleopterous insects collected in the late expedition to the Rocky Mountains, performed by order of Mr. Calhoun, Secretary of War, under the command of Major Long. Journal of the Academy of Natural Sciences of Philadelphia, 3(1823), 298-331.

Selander, R. B. (1981). The caustica group of the genus Epicauta (Coleoptera: Meloidae). Proceedings of the Entomological Society of Washington, 83, 573-591.

Skinner, H. (1904). New Meloidae from Arizona. Entomological News, 15, 217.

Vázquez, J., Madi, Y., Cordero, I. y Rodríguez, B. (2011). Listado de los anfibios del estado Lara, Venezuela. Boletín del Centro de Investigaciones Biológicas, $45,119-212$.

Werner, F. G. (1944). Some new North American species of Epicauta (Coleoptera: Meloidae). Psyche, 50(1943), 65-73.

Werner, F. G. (1957). Epicauta dugesi a valid species (Coleoptera: Meloidae). Psyche, 64, 107-108.

Wuster, W., Yrausquin, J. L. y Mijares-Urrutia, A. (2001). A new species of indigo snake from north-western Venezuela (Serpentes: Colubridae: Drymarchon). Herpetological Journal, 11, 157-166. 